LAWRENCE LIVERMORE NATIONAL LABORATORY

\title{
Estimates of Imaging Times for Conventional and Synchrotron X-Ray Sources
}

J. Kinney

May 20, 2003 
This document was prepared as an account of work sponsored by an agency of the United States Government. Neither the United States Government nor the University of California nor any of their employees, makes any warranty, express or implied, or assumes any legal liability or responsibility for the accuracy, completeness, or usefulness of any information, apparatus, product, or process disclosed, or represents that its use would not infringe privately owned rights. Reference herein to any specific commercial product, process, or service by trade name, trademark, manufacturer, or otherwise, does not necessarily constitute or imply its endorsement, recommendation, or favoring by the United States Government or the University of California. The views and opinions of authors expressed herein do not necessarily state or reflect those of the United States Government or the University of California, and shall not be used for advertising or product endorsement purposes.

\section{Auspices Statement}

This work was performed under the auspices of the U.S. Department of Energy by University of California, Lawrence Livermore National Laboratory under Contract W-7405-Eng-48. 


\title{
SOME NOTES ON 3D-MICROTOMOGRAPHY OF NIF-TARGETS: ESTIMATES OF IMAGING TIMES FOR CONVENTIONAL AND SYNCHROTRON X-RAY SOURCES
}

\author{
J.H. Kinney
}

MMED

December 28, 2002

Contact information:

\section{J.H. Kinney}

(925)-422-6669

kinney3@llnl.gov

Notice: This document provides estimates of the photon fluence required to image a select class of targets using absorption contrast imaging in either parallel or cone-beam geometries. It does not consider phase or diffraction contrast, nor $x$-ray optics like focusing mirrors or condensing lenses. 


\section{BACKGROUND:}

The following notes are to be taken as estimates of the time requirements for imaging NIF targets in three-dimensions with absorption contrast. The estimates ignore target geometry and detector inefficiency, and focus only on the statistical question of detecting compositional (structural) differences between adjacent volume elements in the presence of noise. The basic equations, from the classic reference by Grodzins,(1) consider imaging times in terms of the required number of photons necessary to provide an image with given resolution and noise. The time estimates, therefore, have been based on the calculated x-ray fluxes from the proposed Advanced Light Source (ALS) imaging beamline, and from the calculated flux for a tungsten anode $\mathrm{x}$-ray generator operated in a point focus mode.(5)

\section{THEORETICAL MODEL:}

The theoretical treatment is based upon model proposed by Grodzins.(1) It has been modified to account for an area detector, which allows all of the ray paths to be collected in parallel. Though other, more sophisticated models have been developed, Grodzins' approach has proved to be a good starting point for estimating optimal energies and scan times for microtomography studies.(3) Let $\mu$ be the linear attenuation coefficient(4) of the material composing the target. A normalized variance can be defined in terms of the standard deviation of the attenuation measurement $(\sigma)$ :

$$
\operatorname{var}=\left(\frac{\sigma}{\mu}\right)^{2}
$$

The target is imaged with discrete detector elements (e.g., pixels in a CCD array) of square cross section with area $w^{2}$ ( $w$ is the width of the pixel). The geometry is illustrated in Figure 1. The x-ray source produces a flux of $\phi$ photons per unit area per second at the target. Then the total fluence, $\Phi$, per pixel (summed over all angular views) required to achieve a defined variance in the image is given by:

$$
\phi w^{2} T=\Phi \frac{2 e^{\mu D}}{2 \mu^{2} \text { evar }} \text { this in you con }
$$

In equation 1.2, $T$ is the time required for imaging a target of diameter $D$ at resolution $w$ and variance given by equation 1.1. The time is a minimum when its derivative with respect to $\mu$ is zero, i.e., when $\mu D=2$ :

$$
\Phi_{o p t}=\left(\frac{D}{w}\right)^{2} \frac{e^{2}}{2 \bullet v a r}
$$




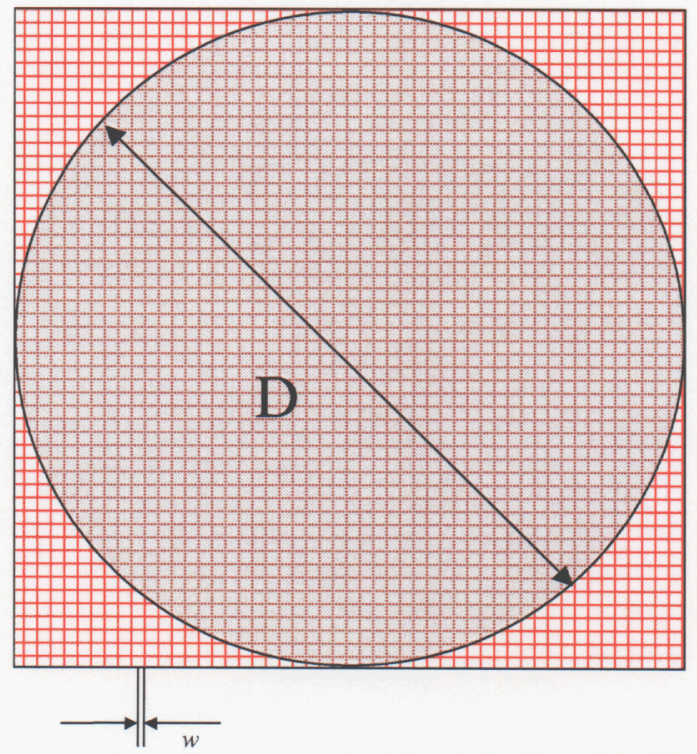

Figure 1: The geometry used to develop order of magnitude estimates for optimizing the detection of flaws in NIF targets of varying composition. The target cross-section of diameter D (shown in gray) is discretized into cubic volume elements (voxels) of size $w$ on an edge.

\section{RESULTS:}

OPTIMAL ENERGIES FOR IMAGING FOAM TARGETS:

For a single composition and thickness, there is an optimal energy that reduces the integration time required to image the specimen. This optimal energy has been calculated for three typical target materials as a function of thickness and density: carbon aerogel, silica aerogel, and tantulum foam (Figure 2). For typical densities, x-rays between $2-10$ $\mathrm{keV}$ will be optimal for most target materials. Perhaps the exception will be Ta foam, whose L-edges below $10 \mathrm{keV}$ create opportunities for imaging with $\mathrm{x}$-rays as high as 30 $\mathrm{keV}$.

For a given target thickness and composition, the fluence required to achieve 5\% noise with $5 \mu \mathrm{m}$ voxels is graphed as a function of $\mathrm{x}$-ray energy in Figure 3 . The behavior of the carbon and silica aerogels is typical of that seen in all thin, low- $Z$ materials: a pronounced minimum in the required fluence at the optimal energy. This behavior, which is less pronounced in the Ta foam, makes the undoped carbon and silica aerogels difficult to image with conventional x-ray sources. For example (Figure 3 ), it takes roughly $5 \times 10^{7}$ photons per pixel area to image a $1-\mathrm{mm}$ diameter carbon aerogel with $5 \mu \mathrm{m}$ resolution and $5 \%$ noise if all of the photons are at the optimal energy of $\sim 2.2 \mathrm{keV}$. If, instead, the $\mathrm{X}$-ray energy is $8 \mathrm{keV}$, the same quality image will require $10^{10}$ photons, or take roughly 200 times longer to image! 

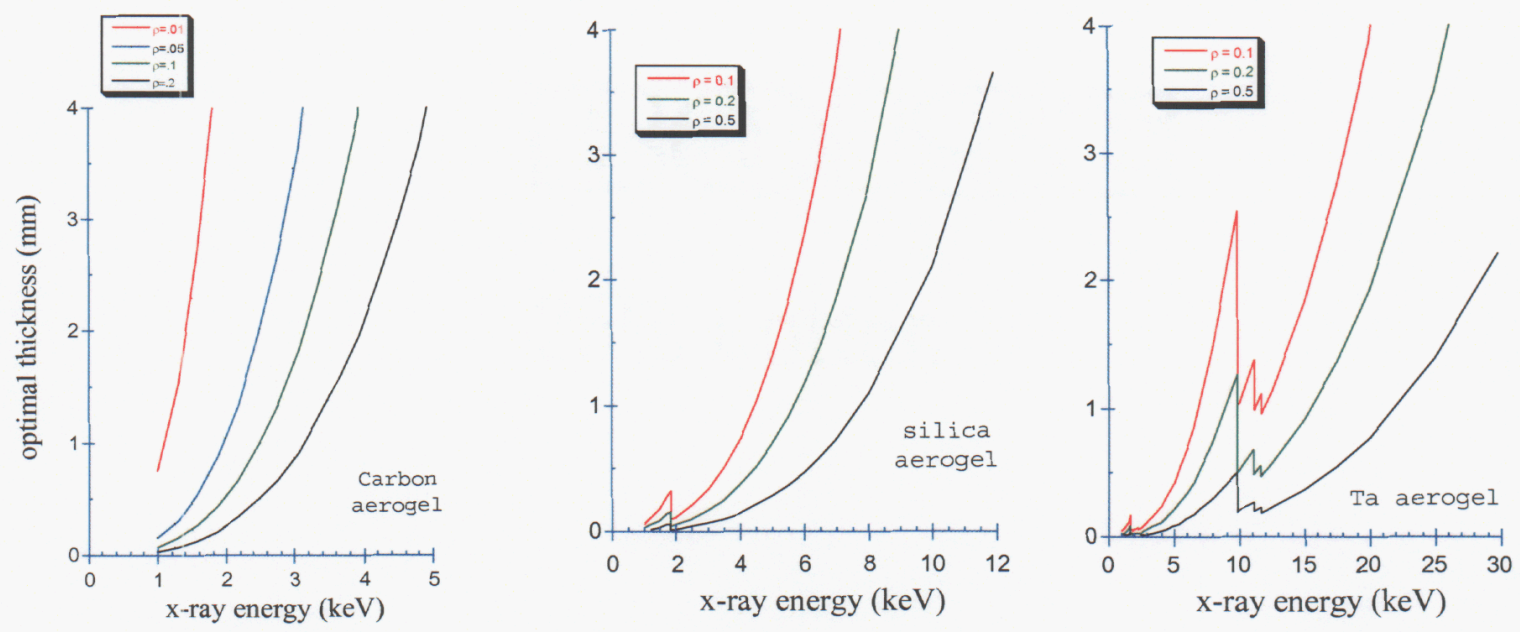

Figure 2: optimal target thickness for a range of $x$-ray energies and material density. For a target thickness less than $2 \mathrm{~mm}$, the carbon and silica aerogels will best be imaged with 2-10 keV x-rays. The exception, Ta, can be imaged effectively either above or below the L-edge.
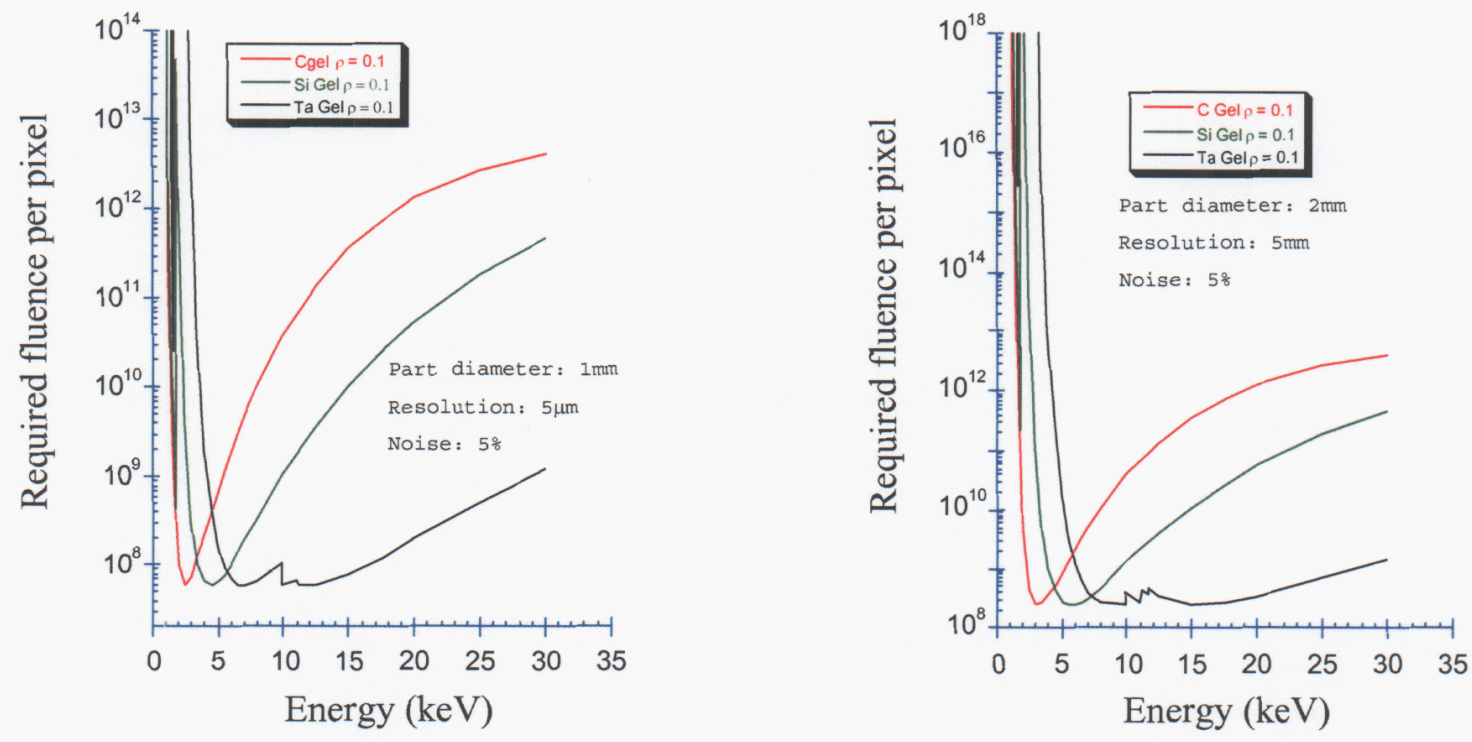

Figure 3: required fluence (photons per square pixel area) for 1-mm (left) and 2-mm (right) diameter targets for carbon and silica aerogels and Ta foam. Density is assumed to be $100 \mathrm{mg} / \mathrm{cc}$; resolution is $5 \mu \mathrm{m}$ and noise is $5 \%$. 

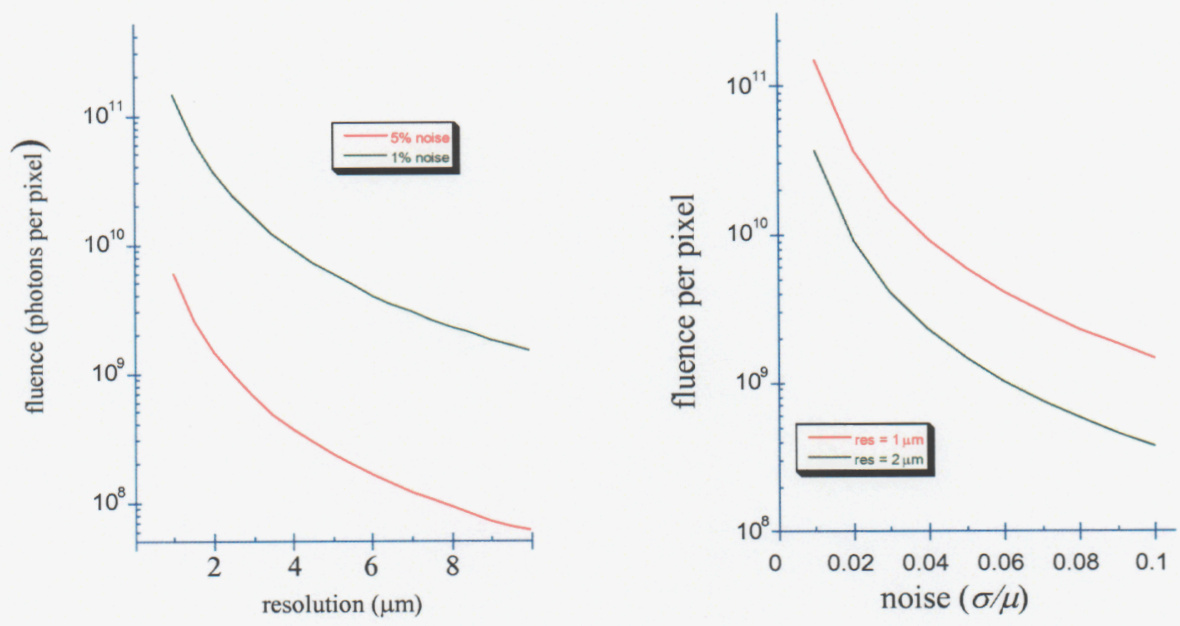

Figure 4: For a given size target, the fluence depends strongly on both the resolution and the noise that can be tolerated in the image. The left graph shows the fluence required to image a $2 \mathrm{~mm}$ diameter target with $1 \%$ and $5 \%$ noise at different resolving powers. A similar graph (right) shows the same dependence for a $2 \mathrm{~mm}$ diameter target. Optimal $x$-ray energy was assumed, so the graphs are independent of the material density (Equation 1.3).

\section{IMAGING TIME WITH SYNCHROTRON RADIATION:}

Calculating the time required to image a specimen at its optimal energy requires good estimates of the source brightness and detector efficiency. For these calculations, we used estimates of the x-ray flux anticipated for a multilayer monochromator on the proposed superbend imaging beamline at the Advanced Light Source (ALS).(2) Because the multilayer is continuously tunable from $2-30 \mathrm{keV}$, we assume optimal energies can be used for each target. Therefore, time estimates can be obtained independent of the target material with Equation 1.3.

The time required to image a $2-\mathrm{mm}$ diameter target with $1 \%$ and $5 \%$ noise levels is graphed as a function of resolving power in Figure 5. Apparent in this figure is the strong penalty in integration time that is excised by increasing the resolving power. For a given source brightness, imaging time scales as $D^{2} / w^{4}$; for the same target diameter and noise, doubling the resolving power requires 16 times longer integration time. If a practical time is about 500 minutes ( $~ 8 \mathrm{hrs})$, then it is not likely even with synchrotron radiation to achieve better than $2-\mu \mathrm{m}$ resolution in $3 \mathrm{D}$ at $5 \%$ noise in a $2-\mathrm{mm}$ diameter target. However, if noise limits are relaxed, or the target size is reduced, it is quite likely that $0.5-1.0-\mu \mathrm{m}$ resolving power can be routinely achieved at a synchrotron light source. 


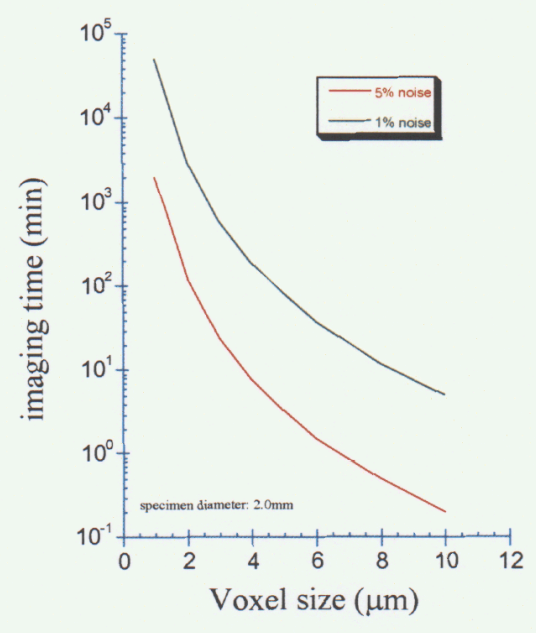

Figure 5: Estimate of the time required to image a $2 \mathrm{~mm}$ diameter target at $1 \%$ and $\mathbf{5 \%}$ noise levels for various resolving powers at the optimal photon energy.

\section{IMAGING TIME WITH POINT FOCUS LABORATORY SOURCE}

Estimating the time required to image a target with a point focus laboratory source is more difficult than estimating time required for a synchrotron source. First, the lab source cannot efficiently be made monochromatic, so the inefficiencies of a polychromatic source must be considered. Second, there are design considerations that govern how close the specimen can be to the source, and how much power can be applied to the anode (heat load dissipation). Finally, there are artifacts in the reconstructed image created by a polychromatic source (beam hardening) that also affect the noise levels in the reconstructed image. Therefore, for the purpose of this brief note, beam hardening has been ignored and geometry and power load have been fixed (these last two parameters easily can be scaled to an actual system).

Because there is pronounced energy sensitivity in the fluence required to image the carbon and silica aerogels, it is likely that 3D imaging with a laboratory source only will be practical in the higher- $Z$ or doped targets. Therefore, the time estimate for a laboratory source is derived for a $2-\mathrm{mm}$ diameter, $0.4 \mathrm{~g} / \mathrm{cc}$ Ta foam target. Image resolution is $5-\mu \mathrm{m}$ and noise is $5 \%$.

The $\mathrm{x}$-ray spectrum for a $100 \mathrm{kVp}, 1-\mathrm{mA}$ tungsten anode source with a 1-mm thick Al filter was calculated from a semi-empirical model developed by Tucker et al.(5) The spectrum, normalized to the maximum differential flux, is graphed in Figure 6. At an object located $20-\mathrm{mm}$ from the source, the integrated x-ray flux is estimated to be $9 \times 10^{9}$ photons $/ \mathrm{mm}^{2} / \mathrm{sec}$. Not all of these photons, however, are equally efficient in their contribution to the signal to noise. Therefore, we define a spectral efficiency factor, $\xi(E)$ : 

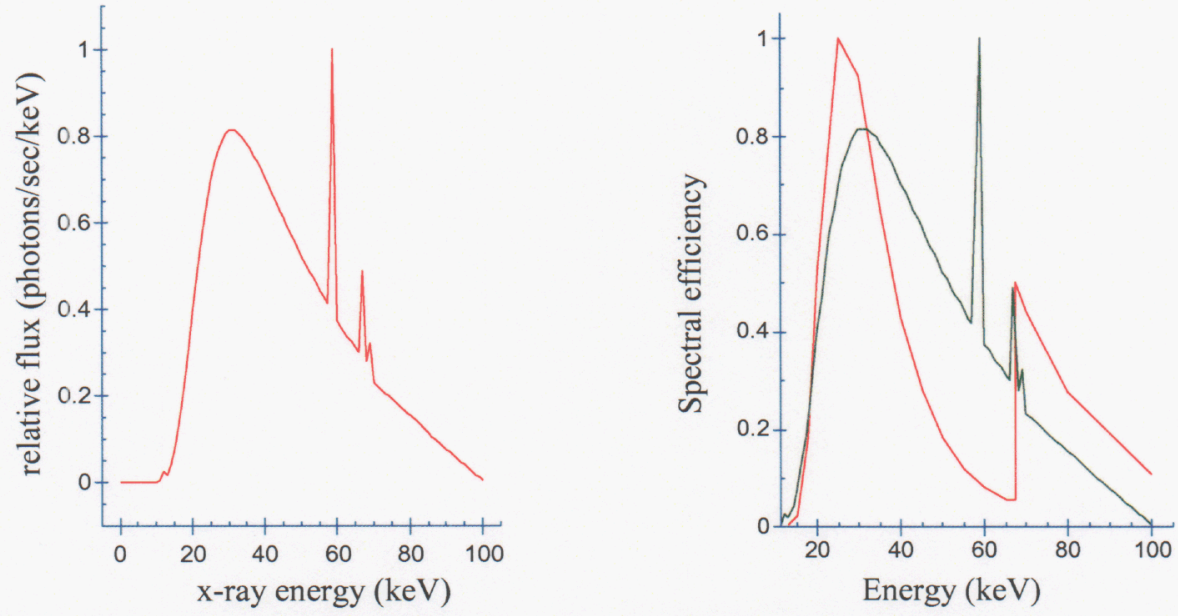

Figure 6: Left: calculated differential $x$-ray flux (relative units) for a point focus Wanode running at $100 \mathrm{kVp}$ and $1-\mathrm{mA}$. A $1-\mathrm{mm}$ thick Al filter has been used to eliminate the contribution to the image from photons less than $10 \mathrm{keV}$. The anode is covered by a $5 \mathrm{~mm}$ thick Be window. Right: superposed on the spectrum is the relative efficiency factor (Equation 1.4). The highest efficiency is at the peak of the Bremsstrahlung spectrum.

$$
\xi(E)=\frac{\Phi_{\text {opt }}}{\Phi(E)}
$$

The average efficiency of the source, $K(E)$, is defined by:

$$
K(E)=\frac{\int_{0}^{k V p} \xi(E) \phi(E) d E}{\int_{0}^{k V p} \phi(E) d E}
$$

For the particular case of the 2-mm diameter Ta foam, integration of equation 1.5 indicates a $50 \%$ spectral efficiency. Thus, a 5- $\mu \mathrm{m}$ image at $5 \%$ noise would take approximately 36 minutes of integration time on a high-power point focus source. Note: this estimate depends critically on the anode current and the distance from the specimen to the source, as well as the accuracy of the calculated $x$-ray spectrum. 


\section{CONCLUSION:}

Synchrotron radiation sources must be considered in any discussion on NIF target characterization. The broad range of target materials and densities requires the ability to accurately select the x-ray energy prior to imaging, a procedure that is impractical with point focus laboratory sources. The exceptions might be targets composed largely of Ta foams, where a broad minimum in the energy dependence of the signal to noise may make it feasible to use a broad, polychromatic spectrum. However, even with synchrotron radiation sources, it is unlikely that large target diameters can be imaged with submicron resolution without a significant relaxation of the noise requirements. If the primary defect is of high contrast, like a void or crack, then this relaxation might be warranted.

The workhorse of $\mathrm{x}$-ray characterization will most likely be $2 \mathrm{D}$ microscopic imaging, either with a point focus source or with $\mathrm{x}$-ray optics (or both). These imaging needs can be achieved with conventional $x$-ray sources operating with changeable anodes. However, for three-dimensional requirements, such as ruled grating targets, it will be necessary to operate with a high-brightness source, such as a synchrotron. This is unavoidable; statistical arguments require the x-ray fluence to scale as $D^{2} / w^{4}$, where $D$ is the target diameter and $w$ is the resolving power of the instrument.

\section{REFERENCES:}

1. Grodzins L: Optimum energies for x-ray transmission tomography of small samples: Applications of synchrotron radiation to computerized tomography I. Nuclear Instruments and Methods 206:541-546, 1983

2. Howells M: Imaging Beamline at the Advanced Light Source. Private correspondence with JH Kinney. Berkeley, CA, 2002

3. Kinney JH, Nichols MC: X-Ray Tomographic Microscopy (XTM) Using Synchrotron Radiation. Annual Review of Materials Science 22:121-152, 1992

4. Plechaty EF, Cullen DE, Howerton RJ: Tables and Graphs of Photon-Interaction Cross Sections from $0.1 \mathrm{keV}$ to $100 \mathrm{MeV}$ Derived from the LLL EvaluatedNuclear-Data Library. Livermore California, Lawrence Livermore National Laboratory, 1981

5. Tucker D, Barnes G, Chakraborty D: Semiempirical model for generating tungsten target x-ray spectra. Medical Physics 18:211-219, 1991 